\title{
Instruire ou étourdir les élèves? Réflexion critique sur l'idée qu'«il faut varier son enseignement»
}

\author{
Clermont Gauthierio \\ Université Laval, Quebec, Canada
}

Steve Bissonnette ${ }^{\mathrm{ii} i \mathrm{D}}$
Université TÉLUQ, Quebec, Canada

Marie Bocquilloniiid

Université de Mons, Mons, Belgique

\begin{abstract}
Résumé
On remarque souvent dans les écrits dans le domaine de l'éducation une invitation à «varier son enseignement». Si cette expression semble claire à première vue, elle devient vite ambigüe à la suite d'un examen un peu plus approfondi car les critères sur lesquels se base cette variation ne sont pas explicités. Tout se passe comme si varier les activités d'apprentissage est vu comme un principe qui a une valeur en soi, mais il ne constitue pas un principe d'action pertinent car il risque en effet de créer de la confusion en mettant sur le même pied des activités qui peuvent avoir des effets positifs ou négatifs selon les contextes d'enseignement. L'objectif de cet article est de formuler des critères de choix d'activités à la lumière des travaux sur l'enseignement explicite, notamment en ce qui concerne la gestion des apprentissages et la gestion de la classe. Le niveau de compétence des lèves, la complexité de la tâche à accomplir, le temps disponible et l'importance du contenu sont les critères retenus en ce qui concerne la gestion des apprentissages. Le maintien du vecteur d'action renvoie au critère lié à la gestion de la classe.
\end{abstract}

Mots-clés

Varier l'enseignement ; Idées reçues ; Critique ; Enseignement explicite ; Critères.

\section{Instruir ou atordoar os alunos? Reflexão crítica sobre a ideia}

de que «precisamos variar o nosso ensino»

\begin{abstract}
Resumo
Frequentemente, na literatura educacional, há um convite para "variar o ensino de alguém". Embora essa expressão possa parecer clara à primeira vista, ela rapidamente se torna ambígua após um exame mais detalhado, porque os critérios nos quais essa variação se baseia não são explicitados. É como se a variação das atividades de aprendizagem fosse vista como um princípio que tem valor em si mesmo, mas não é um princípio de ação relevante, porque corre o risco de criar confusão ao equacionar atividades que podem ter efeitos positivos ou negativos, dependendo do contexto de ensino. O objetivo deste artigo é formular critérios para a escolha de atividades à luz de trabalhos sobre ensino explícito, em particular no que diz respeito à gestão da aprendizagem e à gestão
\end{abstract}

Educ. Form., Fortaleza, v. 6, n. 3, e5404, set./dez. 2021

DOI: https://doi.org/10.25053/redufor.v6i2.5404

https://revistas.uece.br/index.php/redufor/index 
da sala de aula. O nível de competência dos alunos, a complexidade da tarefa a realizar, o tempo disponível e a importância do conteúdo são os critérios utilizados na gestão da aprendizagem. A manutenção do vetor de ação refere-se ao critério relacionado ao gerenciamento da aula.

\title{
Palavras-chave
}

Variedade o ensino; Preconceitos; Crítico; Ensino explícito; Critério.

\section{Instruct or stun the students? Critical reflection on the idea that "we need to vary our teaching"}

\begin{abstract}
Often in the educational literature there is an invitation to "vary one's teaching". While this expression may seem clear at first glance, it quickly becomes ambiguous upon closer examination because the criteria on which this variation is based are not made explicit. It is as if varying learning activities is seen as a principle that has value in itself, but it is not a relevant principle of action because it risks creating confusion by equating activities that may have positive or negative effects depending on the teaching context. The objective of this article is to formulate criteria for choosing activities in the light of work on explicit teaching, particularly regarding learning management and classroom management. The level of competence of the students, the complexity of the task to be accomplished, the time available and the importance of the content are the criteria used regarding learning management. The maintenance of the action vetor refers to the criterion related to the management of the class.
\end{abstract}

\section{Keywords}

Vary the teaching; Misconceptions; Critical; Explicit teaching; Criteria.

\section{Introduction}

Dans une revue récente de la littérature, Dupont et Bouchat (2020) ont examiné les effets sur les performances des élèves originaires de milieux défavorisés de trois orientations pédagogiques recommandées en Belgique francophone via le décret «Missions », à savoir l'approche par compétence, la pédagogie de la découverte et la différenciation. L'approche par compétence renvoie, pour ces auteurs, à la diminution des connaissances factuelles dans les référentiels au profit des compétences. La pédagogie de la découverte, quant à elle, privilégie des activités par lesquelles les élèves découvrent l'objet d'apprentissage par eux-mêmes. Enfin, la différenciation est définie comme «[...] une démarche d'enseignement qui consiste à varier les méthodes pour tenir compte de l'hétérogénéité des classes ainsi que de la diversité des modes et des besoins

Educ. Form., Fortaleza, v. 6, n. 3, e5404, set./dez. 2021

DOI: https://doi.org/10.25053/redufor.v6i2.5404

https://revistas.uece.br/index.php/redufor/index 
d'apprentissage " (COMMUNAUTÉ FRANÇAISE DE BELGIQUE, 1997, article 5). Ces orientations pédagogiques ne sont pas propres à la Belgique francophone et sont semblables à celles préconisées par les réformes d'orientation socioconstructiviste menées depuis une trentaine d'années notamment au Québec, en France et en Suisse.

$\mathrm{Au}$ terme de leur synthèse de la littérature, Dupont et Bouchat (2020, p. 1) indiquent : « (1) rien ne prouve la plus-value des approches [citées précédemment] [...] ; (2) leur application naïve risque d'abaisser le niveau général des élèves et (3) d'augmenter les écarts de performance entre les élèves issus de milieux favorisés et défavorisés ». En plus d'être en accord avec de nombreuses recherches empiriques et synthèses de recherches (par exemple, la méga-analyse de Bissonnette et al. [2010] ou encore la synthèse de Kirschner, Sweller et Clark [2006]), ces propos représentent une contribution assez rare dans la francophonie. En effet, en questionnant le fait que certaines recommandations politiques ne reposent sur aucune donnée probante, ce texte constitue un pas en avant vers une plus grande prise en compte des recherches rigoureuses en éducation.

Paradoxalement, après avoir présenté de nombreuses recherches démontrant la faible efficacité des trois approches pédagogiques citées précédemment, ces mêmes chercheurs concluent en indiquant:

\footnotetext{
En ce qui concerne la pédagogie, nous défendons une approche variée et pertinente. Si l'on veut par exemple amener les élèves à être critiques sur un sujet, il faut que ceux-ci assimilent des connaissances mais aussi qu'ils apprennent à disséquer des arguments, à se poser des questions, à être sceptiques. Et cela ne sera possible que si le professeur utilise différentes pédagogies : de l'explicite, des projets (dans la lignée de la découverte guidée), des enquêtes (mais avec "des balises ") et du questionnement "socratique " (dans le sens de la réfutation). (BISSONNETTE et al., 2010, p. 17, nous soulignon).
}

Pourquoi ces auteurs concluent-ils qu'il faut "varier les pédagogies ", sans fournir de critères pour choisir entre ces dernières dans telle ou telle situation, après avoir démontré l'inefficacité (et l'iniquité) sur l'apprentissage des élèves de certaines? Cela s'explique probablement en partie par la forte aspiration à la liberté pédagogique en Belgique francophone, garantie par la Constitution de 1831. Celle-ci implique notamment le libre choix, par chaque enseignant, des approches pédagogiques qu'il décide de mettre en place dans sa classe. En dehors du contexte belge francophone, l'invitation à 
"varier les pédagogies » est également courante dans le domaine de l'éducation (CFORP, 2016; MEES, 2016) où l'on préfère généralement adopter une formule socialement acceptable auprès des différents acteurs, plutôt qu'affirmer la plus grande efficacité de certaines approches pédagogiques.

Loin de nous aider à mieux comprendre et à agir de manière plus pertinente, ce truisme nous rend plutôt perplexes par son ambiguïté, et partant ne constitue pas une piste d'action féconde d'autant qu'on ne sait pas pourquoi il faudrait varier ${ }^{1}$, ni quoi varier. À preuve, deux confusions dans cette citation se succèdent.

D'abord, si un enseignant veut, comme il est mentionné, développer l'esprit critique chez ses élèves, il doit non seulement viser chez eux l'acquisition de connaissances déclaratives dans un domaine du savoir, mais aussi leur apprendre comment savoir faire une critique, c'est-à-dire un savoir procédural. Ce n'est donc pas la variation en soi qui prévaut ici, mais plus simplement l'alignement des activités choisies avec les objectifs poursuivis. Varier son enseignement n'est donc pas le bon argument à utiliser au sens où la variété ne serait pas forcément plus avisée que la régularité, il s'agit plutôt d'un problème de cohérence des activités choisies en fonction des objectifs poursuivis.

Ensuite, dans l'extrait mentionné, on met sur le même pied ce qui appartient à des niveaux logiques différents. L'élément d'un tout n'est pas du même niveau que le tout, il en fait partie. Ainsi, une activité d'apprentissage n'est pas du même niveau logique qu'une pédagogie qui en contient forcément plusieurs. Rédiger un journal ou faire une enquête sont des activités alors que l'enseignement explicite ou la pédagogie par découverte sont des approches d'enseignement. Par conséquent, choisir deux activités d'apprentissage est fort différent de choisir deux approches pédagogiques même si en apparence il s'agit simplement du même acte de choisir. Les deux activités (rédiger un journal, faire une enquête) peuvent être effectivement équivalentes pour atteindre un

1 D'autant plus qu'il y a 200 ans, on soutenait le contraire dans La conduite des écoles chrétiennes et on faisait l'éloge de la stabilité dans l'enseignement. Le contraste est même comique comme en témoigne cet exemplaire extrait : «Avec des méthodes uniformes, déterminées, invariables, et bien choisies $10^{\circ}[\ldots]$ les Maîtres fatiguent moins; $2 .^{\circ}$ les progrès des écoliers sont plus faciles et plus prompts; $3 .^{\circ}$ les changements de Maitres n'en apportent pas [de changements] dans les principes, ni dans la manière d'enseigner; 4 - $^{\circ}$ les nouveaux Maîtres ne le paraissent pas dans leur enseignement, puisque non seulement ils enseignent les mêmes choses, mais qu'ils les enseignent de la même manière; $5 .^{\circ}$ les principes s'expriment mieux dans l'esprit des écoliers, ils ont moins de peine à les comprendre ; $6 .^{\circ}$ leurs parents les ayant appris eux-mêmes dans les écoles, peuvent les leur enseigner, et être ainsi d'un grand secours aux Maîtres; 7 . ils en prennent occasion d'estimer les Maîtres, et de s'attacher à eux; $8 .^{\circ}$ l'uniformité, l'invariabilité et la clarté des méthodes dans l'enseignement, affectionne davantage les enfants à la doctrine chrétienne » (1819, p. 308).

Educ. Form., Fortaleza, v. 6, n. 3, e5404, set./dez. 2021

DOI: https://doi.org/10.25053/redufor.v6i2.5404

https://revistas.uece.br/index.php/redufor/index 
objectif donné, mais choisir une approche pédagogique (enseignement explicite, pédagogie par projets, etc.) comporte des conséquences beaucoup plus importantes sur l'apprentissage des élèves. On l'a vu notamment avec le projet Follow Through (WATKINS, 1997), les approches pédagogiques comparées ne se valent pas toutes, elles comportent des activités qui n'ont pas toutes les mêmes effets sur les plans cognitifs ou affectifs. Tant et si bien qu'en choisir une plutôt qu'une autre n'est pas seulement une affaire de préférence subjective comme si elles étaient interchangeables mais, plus profondément, une question d'impact sur les apprentissages des élèves.

Au risque de tout emmêler et de diffuser davantage la confusion, on ne peut donc avaliser la prescription « il faut varier son enseignement " sans proposer au préalable un cadre d'analyse et formuler un certain nombre de distinctions. Pour ce faire, nous proposons de procéder de la manière suivante : 1- d'abord, décrire des caractéristiques de la situation pédagogique, ce qui nous permettra de faire ressortir la tension fondamentale entre stabilité et contingence en enseignement; 2- ensuite, définir la pédagogie et ses deux grandes fonctions de base, la gestion des apprentissages et la gestion de la classe; enfin, aux sections 3 et 4 , proposer des critères de variation des activités d'apprentissage à la lumière des précisions apportées sur ces deux grandes fonctions.

\section{Les caractéristiques de la situation pédagogique}

Le travail enseignant se définit à la fois par sa contingence, mais aussi par une structure dotée d'une certaine stabilité. À l'évidence, l'activité enseignante se caractérise par sa nature contingente. Davantage que la plupart des autres activités professionnelles, elle est soumise à des phénomènes tels que la complexité, l'incertitude, l'instabilité, la singularité et les conflits de valeurs (SCHÖN, 1994). II est en effet impossible pour l'enseignant de prévoir tout ce qui se passera durant sa leçon : un élève demandera pour sortir de la classe, un autre dérangera sa voisine, certains comprendront la matière très rapidement, d'autres auront besoin de plus d'explications. L'enseignant devra prendre des décisions, souvent à partir d'informations incomplètes, et sans pouvoir prédire avec certitude leurs effets.

Cet attribut de contingence, Doyle (1986) l'a fait également ressortir, avec d'autres concepts. Pour lui, le processus d'enseignement usuel dans nos écoles 
comporte six caractéristiques générales qui démontrent de manière non équivoque la complexité du métier d'enseignant: 1- la multidimensionnalité; 2- la simultanéité; 3l'immédiateté; 4- l'imprévisibilité; 5- la visibilité; 6- I'historicité. La multidimensionnalité fait référence à la quantité d'événements et de tâches diverses qui sont accomplies dans les classes; la simultanéité renvoie au fait que plusieurs de ces dimensions arrivent en même temps; l'immédiateté rappelle la rapidité du rythme où ceux-ci se déroulent; l'imprévisibilité se rattache à l'inattendu de ces événements; la visibilité souligne la dimension publique, plus spécifiquement des gestes de l'enseignant face à ses élèves; I'historicité manifeste l'impact de ce geste sur les événements à venir dans la classe.

À lire les caractéristiques de Schön (1994) et de Doyle (1986), on serait porté à penser qu'il n'y a absolument rien de stable dans la situation pédagogique, que tout est fluide, mouvant, incertain. Or, ce n'est pas nécessairement le cas. En dépit de sa nature contingente, la situation pédagogique ne nous est jamais totalement inconnue ou nouvelle ; il y a toujours d'une certaine manière quelque chose qui se répète dans la situation de travail de l'enseignant:

\begin{abstract}
We never find ourselves thrown into an "absolutely unfamiliar» situation. There is always some basis on which to interpret that which outside of established paradigms, simply because we are always situated, located at some already familiar locale. Our educational experience, our past, our traditions, our practical interests, always condition our situation, so that whatever temporary contract or consensus we agree to, whatever new paradigm we invent, it will never be absolutely without precedent. (GALLAGHER, 1992, p. 341).
\end{abstract}

Van der Maren (1990) a identifié huit traits fondamentaux propres à la situation pédagogique et qui illustrent également la présence d'une structure relativement stable : 1- Une personne (adulte) censée savoir; 2- est en contacts réguliers; 3- avec un groupe de personnes (enfants); 4- censés apprendre; 5- dont la présence est obligatoire; 6 - pour leur enseigner; 7- un contenu socialement donné; 8- par une série de décisions prises en situation d'urgence.

De même, à partir d'une perspective différente, Durand (1996) a fait ressortir des contraintes de la situation pédagogique qui imposent une certaine stabilité à l'organisation du travail enseignant. Ces contraintes sont, à peu de choses près, assez semblables d'une classe à l'autre et, par conséquent, structurent les comportements des enseignants. Par exemple, des textes officiels définissent les finalités du système d'éducation et des ordres d'enseignement. Plus encore, ces buts sont précisés et

Educ. Form., Fortaleza, v. 6, n. 3, e5404, set./dez. 2021

DOI: https://doi.org/10.25053/redufor.v6i2.5404

https://revistas.uece.br/index.php/redufor/index 
articulés dans des programmes d'enseignement, organisés la plupart du temps autour des disciplines scolaires. De plus, la durée de l'enseignement est établie à dix mois environ par an et les enfants, dont le nombre est déterminé et la présence à l'école obligatoire, y sont regroupés pour cette période. D'ailleurs, la classe ne désigne pas seulement la nature du regroupement (classe de première année, classe de français, etc.), mais aussi l'espace géographique, c'est-à-dire le local où le collectif d'étudiants se retrouve. Cet espace détermine également le comportement des acteurs qui s'y regroupent, tant par sa délimitation fermée que par les objets matériels qu'il contient : bureaux, tables, ordinateurs, tableaux, etc.

Ainsi, si le contingent et la complexité sont des éléments constitutifs de la situation pédagogique, ils ne doivent pas nous faire oublier qu'il existe également dans la classe une structure de base présentant une certaine stabilité. En effet, depuis plus de trois siècles, ce qu'il est convenu désormais d'appeler la forme scolaire (VINCENT, 1980) s'est peu à peu imposée comme le type d'organisation standard de la classe à l'intérieur de laquelle se déroulent les interactions des acteurs.

Les caractéristiques de la situation pédagogique laissent donc entendre que dans l'activité éducative tout n'est pas que contingence; il existe également un contexte général stable qui structure ce qui se passe dans la classe et détermine aussi les agirs des acteurs, enseignant et élèves.

Partant de là, il s'avère qu'on ne peut se limiter dans nos analyses aux seules réalités contingentes et à la nécessité de tout réinventer à chaque instant. Bien que l'activité éducative soit complexe et multiforme, elle doit être saisie également dans ce qu'elle recèle de stabilité. Cette généralité, ce qui échappe dans une certaine mesure au contingent, est justement ce qu'une base de connaissances en enseignement tend à identifier et à analyser. Plus précisément, la question fondamentale d'une base de connaissances est de savoir s'il est possible, à partir de multiples observations et expérimentations contrôlées, de formaliser certaines stratégies de l'enseignant qui lui permettraient de faire face au contingent de sa situation. En somme, une base de connaissances en enseignement serait possiblement en mesure d'aider l'enseignant à construire un ordre dans la situation complexe et incertaine de la classe.

Educ. Form., Fortaleza, v. 6, n. 3, e5404, set./dez. 2021 


\section{La pédagogie, ses deux grandes fonctions de base et la recherche qui l'alimente}

Nous pouvons définir la pédagogie comme étant l'ensemble des actions que l'enseignant met en œuvre dans le cadre de ses fonctions d'instruction et d'éducation d'un groupe d'élèves dans la classe. Ces comportements de l'enseignant visent à créer et à maintenir un certain ordre pour que l'apprentissage des contenus et l'éducation adviennent. Par " ordre ", on entend une organisation suffisamment structurée et stable pour que des collectifs d'élèves puissent être réceptifs mentalement et affectivement aux contenus que l'enseignant veut leur faire apprendre et aux valeurs qu'il veut leur inculquer.

La première fonction pédagogique, la gestion des apprentissages, renvoie à l'enseignement des contenus. En effet, dans le cadre de son travail, l'enseignant doit couvrir le programme, il doit mettre en place un certain nombre d'activités afin que les divers éléments de la matière soient appris et maîtrisés. La seconde fonction concerne la gestion de la classe: prévenir le désordre, organiser les groupes, établir des règles de vie, réagir aux comportements inacceptables, enchaîner les activités, etc. Là aussi, l'enseignant intervient pour réguler le flot d'activités. Ce sont précisément les deux fonctions pédagogiques fondamentales liées à l'enseignement en salle de classe. Un enseignant efficace est celui qui, maîtrisant ces deux fonctions pédagogiques, facilitera la réussite scolaire des élèves dans sa classe.

Nous avons déjà montré (GAUTHIER et al., 1997) que nous disposons maintenant d'une base de connaissances qui prend appui sur la recherche et qui nous permet désormais de mieux intervenir en gestion des apprentissages et en gestion de la classe. Nous avons également décrit en détail (BISSONNETTE; GAUTHIER; CASTONGUAY, 2016; BISSONNETTE; RICHARD; GAUTHIER, 2005; GAUTHIER; BISSONNETTE; RICHARD, 2013) le contenu d'une approche d'enseignement qui fait partie de cette base de connaissances, l'enseignement explicite.

De manière synthétique, il s'agit d'une approche pédagogique structurée et systématique qui comporte notamment trois grandes étapes²:

2 Pour compléter cette description (trop) synthétique, le lecteur intéressé par cette approche pédagogique peut se référer aux ouvrages de Bissonnette et al. (2016) et Gauthier, Bissonnette et Richard (2013).

Educ. Form., Fortaleza, v. 6, n. 3, e5404, set./dez. 2021

DOI: https://doi.org/10.25053/redufor.v6i2.5404

https://revistas.uece.br/index.php/redufor/index 
1. Le modelage durant lequel l'enseignant présente le contenu d'une façon précise et concise, à l'aide d'exemples et de contre-exemples, en vue de favoriser un niveau de compréhension le plus élevé possible. II « met un hautparleur sur sa pensée » en verbalisant aux élèves les liens qu'il effectue pour comprendre la tâche, les questions qu'il se pose, ainsi que les stratégies qu'il mobilise pour la réaliser ;

2. La pratique guidée durant laquelle les élèves réalisent, en groupes et avec l'enseignant, des tâches semblables à celles qui ont été montrées lors du modelage. Durant cette étape, l'enseignant s'assure de vérifier la compréhension des élèves. Pour ce faire, il veille à interroger régulièrement les élèves durant la réalisation de ces tâches. Deux éléments clés orientent la pratique guidée : d'abord, le questionnement par l'enseignant doit être fréquent et la rétroaction constante, et ensuite, l'exécution d'un nombre suffisant d'exercices doit permettre d'atteindre un seuil élevé de réussite;

3. La pratique autonome, qui permet à l'élève de parfaire sa compréhension jusqu'à l'obtention d'un niveau de maîtrise le plus élevé possible. L'atteinte d'un niveau de maîtrise élevé obtenu grâce aux multiples occasions de pratique permet d'améliorer l'organisation des apprentissages en mémoire à long terme et d'en assurer la fluidité et l'automatisation. L'automatisation facilite leur rétention et leur rappel éventuel et libère ainsi la mémoire de travail qui pourra, éventuellement, se consacrer à des aspects plus complexes d'une tâche d'apprentissage.

L'enseignement explicite se distingue d'autres approches pédagogiques, telles que l'approche par la découverte, notamment en ce qui concerne le moment auquel l'apprenant est confronté à des tâches complexes. Alors que dans l'approche par la découverte, on propose aux élèves des tâches complexes généralement dès le début d'un apprentissage, dans l'enseignement explicite, on s'assure d'abord que l'élève maîtrise toutes les habiletés simples nécessaires à la réussite de la tâche complexe avant de lui soumettre. Lorsque ces habiletés spécifiques sont maîtrisées, l'enseignement explicite cherche à favoriser le transfert en invitant les élèves à les utiliser dans des situations complexes. 
L'enseignement explicite est l'approche pédagogique qui nous servira d'ancrage. C'est à travers l'enseignement explicite et au regard de ces deux composantes de l'enseignement (gestion des apprentissages et des comportements) que nous allons analyser l'idée de varier l'enseignement.

\title{
4 Varier des activités dans la perspective de la gestion des apprentissages
}

Au dix-neuvième siècle, Herbart mettait en scène à sa manière la tension qui existe entre faire découvrir, ce qu'il appelle l'expérience, et faire apprendre, c'est-à-dire l'instruction. On nous pardonnera ce long mais révélateur extrait exprimant l'exaspération de ce pédagogue chevronné face à un jeune sans repère, étourdi en quelque sorte.

\begin{abstract}
L'expérience semble compter que l'instruction la suivra, pour décomposer les masses qu'elle a jetées entassées, pour en rassembler et en ordonner les fragments épars et informes. Quel aspect offre, en effet, la tête d'un homme qui n'a pas reçu l'instruction? On n'y voit ni haut ni bas déterminé, les choses n'y sont pas même rangées dans un certain ordre, tout y flotte pêle-mêle. Les idées n'ont pas appris à attendre. Qu'une occasion se présente, et toutes affluent, autant que le fil de l'association en a mis en mouvement, et autant qu'il en peut tenir à la fois dans la conscience. Celles qui, par des impressions fréquemment répétées, ont acquis le plus de force, se font valoir ; elles attirent à elles ce qui leur convient, et refoulent ce qui les gêne. On regarde le nouveau avec étonnement, ou on le néglige, ou bien on le juge vite par une réminiscence. Rien n'en est détaché s'il ne s'y rapporte ! Le point essentiel n'est pas mis en lumière; ou bien, si d'heureuses dispositions naturelles donnent au regard une bonne direction, les moyens manquent pour suivre la trace trouvée. - C'est ce qui se passe quand on commence à instruire un garçon sans culture de dix à quinze ans. Tout d'abord il est absolument impossible de donner à son attention un cours égal. Comme l'ordre n'est maintenu par aucune idée principale, faute de subordination des idées entre elles, l'âme se jette sans cesse, inquiète, de tous côtés ; à la curiosité succède la distraction, et tout tourne au jeu. (HERBART, 1894, p. 97).
\end{abstract}

Cette tension entre faire découvrir et faire apprendre est encore présente de nos jours. Même si nous pensons que l'enseignement explicite est une approche à privilégier dans l'enseignement, et qu'elle se situe plus du côté du " faire apprendre » que de celui de "faire découvrir ", il reste qu'il n'est pas pour autant essentiel de mobiliser constamment l'ensemble des stratégies que cette approche d'enseignement comporte. Des choix doivent être faits pour ajuster le plus possible les activités au contexte de la classe. C'est particulièrement au niveau de la guidance que ces choix doivent être faits dans la mesure où la pratique guidée y occupe la part de temps la plus importante du travail des élèves.

Educ. Form., Fortaleza, v. 6, n. 3, e5404, set./dez. 2021

DOI: https://doi.org/10.25053/redufor.v6i2.5404

https://revistas.uece.br/index.php/redufor/index 
Quatre critères sont à privilégier par l'enseignant et colorent le choix de ses activités (et des approches pédagogiques sous-jacentes telles que l'enseignement explicite et l'approche par la découverte) : 1. le niveau de compétence des élèves; 2 . le degré de nouveauté et de complexité de la tâche proposée; 3. le temps disponible; 4. les idées maîtresses du curriculum. Ceux-ci sont illustrés dans la figure ci-dessous qui situe sur un continuum le niveau de guidance, de (+) pour l'enseignement explicite à (-) pour l'approche par découverte.

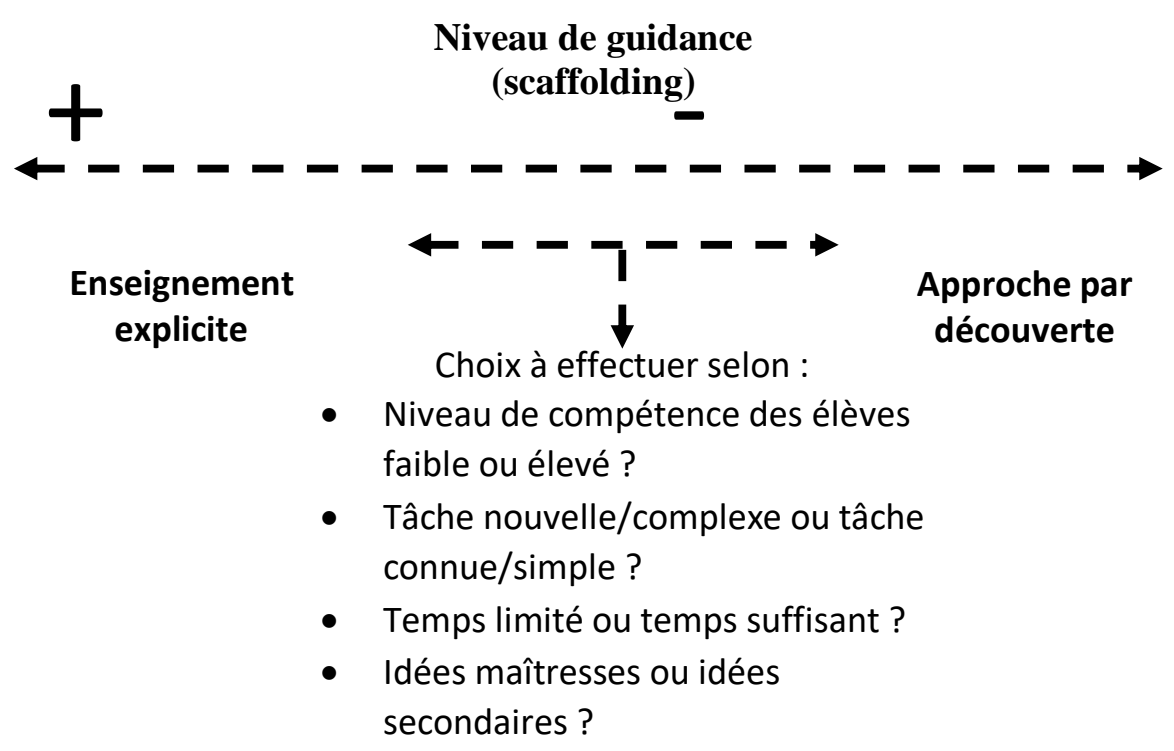

C'est bien connu, le niveau de compétence des élèves varie dans une classe. Lorsque l'enseignant a affaire à un groupe plus faible, il lui sera difficile d'entrée de jeu de leur faire apprendre via des activités fondées sur une approche par la découverte en raison du fait qu'il leur manque les préalables nécessaires pour y arriver et que cette situation risque de les amener à la surcharge cognitive (KIRSCHNER; SWELLER; CLARK, 2006). Ces élèves ont besoin d'activités fondées sur un enseignement explicite qui favorise leur progression pas à pas en respectant les limites de leur mémoire de travail (SWELLER; KIRSCHNER; CLARK, 2007). En revanche, certains groupes ont un niveau de compétence plus élevé et leur niveau ne requiert pas de commencer une séquence d'apprentissage à partir de zéro. Les élèves possèdent alors les préalables pour réaliser avec succès des activités présentant un certain niveau de découverte.

Par ailleurs, une tâche peut être connue et/ou encore simple, elle peut également être nouvelle et/ou complexe. Le choix des activités d'apprentissage ne sera pas le 
même dans ces cas et ce qui est considéré comme nouveau et/ou complexe nécessitera un étayage plus soutenu (comme le propose l'enseignement explicite) que pour des tâches simples et/ ou connues.

Le temps dont l'enseignant dispose influencera aussi le choix des activités qu'il peut proposer aux élèves. On sait que des activités basées sur la découverte demandent plus de guidance pour les élèves moins forts et par conséquent nécessiteront un investissement en temps plus considérable.

Enfin, le contenu peut avoir une importance première ou moindre. Dans ce cas, on parlera d'idées maîtresses ou secondaires. Les idées maîtresses sont au curriculum ce que les idées principales sont au texte informatif : «[...] les idées maîtresses renvoient aux éléments centraux, aux principes, aux concepts clés, aux stratégies heuristiques autour desquels l'enseignement de plusieurs autres contenus ou habiletés d'apprentissage peut être rattaché et organisé "(GAUTHIER et al., 2013, p. 106). L'enseignement des différentes structures de texte (narrative, informative, nouvelle journalistique, etc.) dans un programme de français représente un bon exemple d'idées maîtresses. Étant donné l'importance et la complexité des idées maîtresses, celles-ci doivent faire l'objet d'un enseignement explicite à travers les étapes du modelage, de la pratique guidée et de la pratique autonome, et ce, pour permettre à tous les élèves (et pas seulement aux plus forts) de les acquérir.

En résumé, lorsque l'enseignant s'adresse à des élèves dont le niveau de compétence est faible par rapport aux objets d'apprentissage, que plusieurs de ceux-ci éprouvent des difficultés, que la tâche qu'il leur propose est nouvelle ou complexe, que le temps disponible est limité, qu'il s'agit de l'enseignement d'une idée maîtresse, il convient alors d'utiliser la démarche de l'enseignement explicite. Ces mégacritères, liés spécifiquement à la gestion des apprentissages, permettront à l'enseignant de choisir des activités pertinentes pour ses élèves, et ce, en fonction du degré de guidance qu'il estime nécessaire. Plusieurs activités fondées sur diverses approches pédagogiques peuvent être proposées par l'enseignant, mais ces quatre critères permettent d'en faire une sélection raisonnée et moins subjective.

Inversement, et ce n'était visiblement pas le cas de l'élève dont parlait Herbart, lorsque l'enseignant s'adresse à des élèves dont le niveau de compétence est élevé, que la tâche proposée est connue ou simple et que le temps disponible est suffisant, il est 
possible d'utiliser dans ce contexte des approches par découverte où le niveau de guidance requis est minimal. Les idées secondaires, quant à elles, peuvent éventuellement être enseignées via une approche par la découverte, mais uniquement si le degré de compétence des élèves est élevé, si la tâche est simple/connue et si le temps disponible est suffisant.

En somme, et c'est sans doute ici que se situe le point crucial : la question n'étant pas de varier à tout prix les activités et les approches pédagogiques mais bien de les varier, si le besoin de guidance le nécessite, et ce, en fonction des quatre critères mentionnés. II importe donc pour l'enseignant d'ajuster le niveau de guidance des activités d'apprentissage qu'il propose aux élèves selon ces éléments et non pas sur la base superficielle d'idées reçues du type « il faut varier les activités et les approches pédagogiques ».

\section{Varier des activités dans la perspective de la gestion de la classe}

La seconde grande fonction de l'enseignement, la gestion de la classe, vise à réguler le flot des activités, c'est-à-dire à maintenir un certain ordre pour que l'instruction et l'éducation puissent advenir. Non pas l'ordre au sens abusif du terme, mais plutôt une certaine forme d'ordre fonctionnel pour que, dans le cadre d'un travail en collectif comme celui de la classe, des apprentissages puissent se réaliser.

Order does not necessarily mean passivity, absolute silence, or rigid conformity to rules, although these conditions are sometimes considered necessary for specific purposes (e.g. a major test). Order in a classroom simply means that within acceptable limits the students are following the program of action necessary for a particular classroom event to be realized in the situation. (DOYLE, 1986, p. 396, nous soulignons).

Cette idée de programme ou de vecteur d'action nous semble essentielle. On pourrait décrire ce concept comme regroupant les activités d'apprentissages que l'enseignant cherche à installer, à maintenir ou à restaurer dans sa classe.

Les travaux de Kounin (1970) ont révélé que les différences importantes entre le succès et l'insuccès des enseignants à gérer la classe ne se situent pas tant sur le plan de leurs réactions à l'inconduite de leurs élèves même s'il est important évidemment d'y réagir. Elles se situent plutôt dans leur capacité à prévenir, à anticiper l'apparition 
d'écarts de conduite, et ce, contrairement aux stratégies correctives utilisées une fois que ces problèmes se sont produits. Par diverses stratégies les enseignants efficaces réussissent donc à prévenir les ruptures du programme d'action (les activités d'apprentissage) qu'ils ont installé dans leur classe. Le vecteur principal d'action dans lequel sont engagés les élèves avec l'enseignant peut parfois entrer en conflit avec d'autres vecteurs d'actions (dans ce cas-ci des comportements perturbateurs) que désireraient mettre en place un ou plusieurs élèves qui résistent et veulent faire autre chose que l'activité demandée. L'enseignant devra alors intervenir tout en interférant le moins possible avec le flux du vecteur principal. L'élément premier à considérer en ce qui concerne la gestion de la conduite des élèves au regard des activités d'apprentissage est donc d'anticiper les écarts de conduite avant qu'ils n'apparaissent afin de maintenir le vecteur d'action qui a été préalablement installé. L'enseignant devra anticiper les éléments risquant de diminuer la fluidité du vecteur d'action.

Ces quelques précisions sur la gestion de la classe nous permettent de jeter un autre éclairage sur l'idée de varier les activités. En fait, dans une perspective de gestion de la classe, l'enseignant aura d'abord en tête le maintien de son vecteur d'activités. Par exemple, dans un cas de figure comme la fatigue ou encore la surcharge cognitive, il pourra estimer nécessaire d'interrompre une activité soutenue et/ou la remplacer par une autre quand il commence à percevoir chez ses élèves qu'un certain niveau de saturation est atteint. II y a longtemps, Herbart (1894, p. 141, nous soulignons) soulignait cet aspect de la manière suivante :

Mais l'enseignement fatigue en général d'autant plus vite qu'il est donné plus longtemps d'une façon suivie, bien qu'à cet égard il y ait des différences d'un élève à l'autre. Or, plus il fatigue les élèves, moins il donne de résultats comme occupation. Ceci suffit à montrer la nécessité d'interrompre de temps à autre les occupations et de les varier.

En fait, il s'agit là d'une variation dictée tout simplement par le savoir d'expérience d'un enseignant perspicace qui perçoit bien que les élèves ont suffisamment travaillé et ont besoin d'une pause. S'entêter et ne pas tenir compte de cette observation causerait sans aucun doute l'apparition de problèmes de comportements dans la classe. II faut donc prévenir ces écarts de conduite potentiels. Un autre cas de figure est la monotonie et celle-ci devient un problème quand elle risque de perturber le programme d'action. À nouveau, comme le signalait Herbart (1894, p. 96) de 
manière plutôt péremptoire, "Être ennuyeux, c'est le péché le plus grave que puisse commettre quiconque se mêle d'enseigner". Personne ne peut cependant garantir, malgré ses efforts, qu'il ne sera pas à son tour ennuyeux, mais la question importante est ailleurs, quand le maître perçoit que son vecteur d'action est menacé, il devra varier son enseignement. S'il ne l'est pas, même si l'activité est routinière, comme lire un livre en entrant dans la classe, il n'y a aucun intérêt à changer. Certaines activités, précisément parce qu'elles sont routinières, sécurisent les enfants et préviennent les écarts de conduite (BISSONNETTE; GAUTHIER; CASTONGUAY, 2016).

II arrive que la prévention ne soit pas suffisante et que l'enseignant doive intervenir de manière à faire cesser les comportements perturbateurs. Dans ce cas, il faut distinguer un écart de conduite mineur d'un écart de conduite majeur. Un écart de conduite mineur est un manquement aux attentes comportementales préalablement enseignées qui ne nuit pas au bon fonctionnement de la classe ni à l'apprentissage des élèves, donc qui ne remet pas en question le vecteur d'activité, mais dérange l'élève lui-même ou quelques élèves autour de lui (BISSONNETTE; GAUTHIER; CASTONGUAY, 2016). Dans ce cas, l'enseignant pourra recourir en premier lieu à des interventions indirectes comme: contrôler par la proximité, donner des directives non verbales, ignorer intentionnellement et renforcer de manière différenciée, etc. $\mathrm{Si}$ le comportement perturbateur persiste, l'enseignant pourra opter pour des interventions plus directes comme: rediriger l'élève, réenseigner le comportement attendu, offrir un choix à l'élève, recourir aux conséquences formatives, rencontrer l'élève individuellement, etc. Tout ceci est fait dans le but de maintenir en place le vecteur d'action et de ne pas interférer avec le flux des activités.

Par ailleurs, un écart de conduite majeur est soit un manquement aux attentes comportementales préalablement enseignées qui nuit au bon fonctionnement de la classe, à l'enseignement du maître et, par conséquent, à l'apprentissage des autres élèves, soit un comportement dangereux, illégal ou illicite. Dans ce cas, vu l'ampleur du problème, il est impossible pour le maître de maintenir son vecteur d'activité. Celui-ci doit donc être restauré. L'enseignant opte alors pour des dispositifs plus costauds. II peut, par exemple, s'avérer nécessaire de faire appel à de l'aide spécialisée (psychoéducateur, éducateur spécialisé, etc.) pour venir l'appuyer dans la classe ou prendre en charge l'élève en dehors de la classe. 
En résumé, sur le plan de la gestion de la classe, l'enjeu n'est pas forcément la variation des activités « en soi », mais plutôt d'instaurer et de maintenir la stabilité dans le fonctionnement de la classe afin de permettre au vecteur d'action de l'enseignant de produire ses effets au maximum. Sur le plan de la gestion de la classe, la majeure partie des interventions, $80 \%$, sera consacrée à la prévention de l'apparition des écarts de conduite en vue du maintien du vecteur d'action et $20 \%$ des interventions viseront à corriger les écarts de conduite afin de maintenir, si les problèmes sont mineurs ou de restaurer, si les problèmes sont majeurs, le vecteur d'activité établi par l'enseignant.

\section{Conclusion}

Nous ne sommes pas nostalgiques de l'époque des Frères des écoles chrétiennes qui valorisaient la stabilité avant tout, comme nous ne sommes pas non plus des apôtres de la variété à tout prix. Varier les activités d'apprentissage n'est pas un principe qui a une valeur en soi.

Notre thèse est ailleurs. Quand les occasions d'apprentissage sont présentes et que les élèves sont engagés dans leur travail, la situation est donc stable et on n'a donc pas de raisons de changer ce qui fonctionne bien. Par contre, si l'enseignant perçoit qu'un changement du niveau de guidance est nécessaire sur le plan de la gestion des apprentissages, il peut alors analyser les activités proposées en fonction des quatre critères mentionnés. Sur le plan de la gestion de la classe, c'est en examinant ce qui empêche le vecteur d'enseignement de se déployer qu'il peut aussi rectifier le tir. L'enseignant variera donc les activités d'apprentissage s'il perçoit des indications dans le comportement des élèves indiquant que le niveau de guidance ne convient pas ou encore s'il ne réussit pas à maintenir le vecteur d'activité proposé. Ces deux cas de figure vont de pair.

C'est sans doute la raison pour laquelle Slavin (2014) affirme deux idées qui semblent contraires à première vue. D'une part, il indique que l'approche de gestion de classe la plus efficace est celle qui consiste à avoir un enseignement des contenus efficace $^{3}$. D'autre part, il affirme aussi qu'un bon enseignement des contenus signifie une

${ }^{3}$ « The most effective approach to classroom management is effective instruction » (SLAVIN, 2014, p. 67).

Educ. Form., Fortaleza, v. 6, n. 3, e5404, set./dez. 2021

DOI: https://doi.org/10.25053/redufor.v6i2.5404

https://revistas.uece.br/index.php/redufor/index 
bonne gestion de classe ${ }^{4}$. Ce qui semble à première vue une contradiction n'est peut-être en réalité qu'une manière d'illustrer l'interdépendance entre les deux grandes fonctions de l'enseignement. Un enseignement des contenus efficace est celui qui, par les activités choisies, réussit à offrir aux élèves le niveau de guidance approprié et partant, une gestion de classe sans problème, de même une bonne gestion de classe est celle qui donne la possibilité à l'enseignant d'installer, de maintenir ou de restaurer un environnement d'apprentissage approprié. Ce sont là les deux faces d'une même médaille.

À défaut de prendre en considération ces éléments, varier son enseignement pour le varier, c'est contribuer à produire le type d'élève que fustigeait Herbart (1894), un être déboussolé sans repères qui se promène dans toutes les directions, autrement dit, un étourdi.

\section{Références}

BISSONNETTE, S. et al. Quelles sont les stratégies d'enseignement efficaces favorisant les apprentissages fondamentaux auprès des élèves en difficulté de niveau élémentaire ? Résultats d'une méga-analyse. Revue de recherche appliquée sur l'apprentissage, v. 3, n. 1, p. 1-35, 2010.

BISSONNETTE, S.; GAUTHIER, C.; CASTONGUAY, M. L'enseignement explicite des comportements. Pour une gestion efficace des élèves en classe et dans l'école. Montréal : Chenelière Éducation, 2016.

BISSONNETTE, S.; RICHARD, M.; GAUTHIER, C. Échec scolaire et réforme éducative. Quand les solutions proposées deviennent la source du problème. Québec : PUL, 2005.

CFORP. Varier les stratégies d'enseignement et d'apprentissage à haut rendement. 2016.

COMMUNAUTÉ FRANÇAISE DE BELGIQUE. Décret définissant les missions prioritaires de l'enseignement fondamental et de l'enseignement secondaire et organisant les structures propres à les atteindre (24 juillet 1997). Moniteur Belge, 23 sept. 1997, p. 24653.

DOYLE, W. Classroom organization and management. In: WITTROCK, M. C. (dir.). Handbook of research on Teaching. New York : Macmillan, 1986. p. 392-431.

${ }^{4}$ C'est précisément le titre de son article: «Good instruction is good classroom management ».

Educ. Form., Fortaleza, v. 6, n. 3, e5404, set./dez. 2021

DOI: https://doi.org/10.25053/redufor.v6i2.5404

https://revistas.uece.br/index.php/redufor/index 
DUPONT, S.; BOUCHAT, P. Lorsque la psychologie cognitive s'intéresse au Décret Missions : Constats et recommandations. Les cahiers du GIRSEF, n. 118, p. 1-21, 2020.

DURAND, M. L'enseignement en milieu scolaire. Paris : PUF, 1996.

FRÈRES DES ÉCOLES CHRÉTIENNES. La Conduite des écoles chrétiennes. Lyon : Rusand, 1823.

GALLAGHER, S. Hermeneutics and education. Albany : State University of New York, 1992.

GAUTHIER, C. et al. Enseignement explicite et réussite des élèves. La gestion des apprentissages. Montréal : ERPI, 2013.

GAUTHIER, C. et al. Pour une théorie de la pédagogie. Recherches contemporaines sur le savoir des enseignants. Québec: l'Université Laval et Éditions De Boeck, 1997.

HERBART, J. F. Principales œuvres pédagogiques. Traduit par A. Pinloche. Travaux et mémoires des Facultés de Lille, Tome 14, mémoire numéro 15. Lille : Félix Pécan, 1894.

KIRSCHNER, P. A.; SWELLER, J.; CLARK, R. E. Why minimal guidance during instruction does not work: an analysis of the failure of constructivist, discovery, problembased, experiential, and inquiry-based teaching. Educational Psychologist, v. 41, n. 2, p. 75-86, 2006.

KOUNIN, J. S. Discipline and group management in classrooms. New York : Holt, Rinehart and Winston, 1970.

MEES. Complément au programme de formation de l'école québécoise. Enseignement primaire et secondaire. Français, langue seconde. La différenciation pédagogique. Québec : Gouvernement du Québec, 2016.

SCHÖN, D. A. Le praticien réflexif. À la recherche du savoir caché dans l'agir professionnel. Traduction de J. Heynemand et D. Gagnon. Montréal : Logiques, 1994.

SLAVIN, R. Good instruction is good classroom management. In: SLAVIN, R. (ed.). Classroom management and assessment. Californie : Corwin, 2014. p. 66-70.

SWELLER, J.; KIRSCHNER, P. A.; CLARK, R. E. Why minimally guided teaching techniques do not work: a reply to commentaries. Educational Psychologist, v. 42, n. 2, p. 115-121, 2007.

VAN DER MAREN, J.-M. Les savoirs et la recherche pour l'éducation. In : ROY, G. R. (dir.). Contenus et impacts de la recherche universitaire actuelle en sciences de l'éducation. Actes du $2 e$ congrès des sciences de l'éducation de langue française du

Educ. Form., Fortaleza, v. 6, n. 3, e5404, set./dez. 2021

DOI: https://doi.org/10.25053/redufor.v6i2.5404

https://revistas.uece.br/index.php/redufor/index 
Canada, tome 3. L'enseignement et l'apprentissage. Sherbrooke : CRP, 1990. p. 10231031.

VINCENT, G. L'école primaire française. Étude sociologique. Lyon : PUL, 1980.

WATKINS, C. L. Project follow through: a case study of contingencies influencing instructional practices of the educational establishment. Cambridge: Cambridge Center for Behavioral Studies, 1997.

Clermont Gauthier, Université Laval, Département d'études sur l'enseignement et l'apprentissage iDhttps://orcid.org/0000-0003-1998-7506

Ph.D., est professeur associé à la Faculté des sciences de l'éducation de l'Université Laval. Ses recherches portent sur l'évolution de l'enseignement, les courants pédagogiques, l'enseignement explicite et la formation des enseignants. II a publié, seul ou en collaboration, plus d'une quarantaine d'ouvrages dont plusieurs ont été traduits en portugais.

Contribution d'auteur: Conception et rédaction.

E-mail: clermont.gauthier@fse.ulaval.ca

\section{Steve Bissonnette, Université TÉLUQ, Département et Programme, Département Éducation}

ii(Dhttps://orcid.org/0000-0002-6340-6062

Ph.D., est professeur au Département d'éducation à la TÉLUQ. II s'intéresse aux travaux sur l'efficacité de l'enseignement et des écoles, à l'enseignement explicite, à la gestion efficace des comportements ainsi qu'aux approches pédagogiques favorisant la réussite des élèves en difficulté.

Contribution d'auteur: Analyse de documents, relecture approfondie et discussions.

E-mail: steve.bissonnette@teluq.ca

Marie Bocquillon, Universités, département et programme Université de Mons, Institut
d'Administration scolaire
iii'@inttps://orcid.org/0000-0002-0518-1539
Assistante au sein de l'Institut d'Administration scolaire de l'Université de Mons (Belgique), intervient
dans le cadre de la formation des futurs enseignants du secondaire supérieur et des étudiants inscrits
au Master en Sciences de l'Education. Sa thèse porte sur le développement d'un outil d'observation
des gestes professionnels et d'aide à l'analyse réflexive au regard de la littérature sur l'enseignement
efficace et l'enseignement explicite. Elle dispense également des formations continuées relatives à
l'enseignement explicite des contenus et des comportements dans des écoles primaires et
secondaires.
Contribution d'auteur: Recension d'écrits, relecture approfondie et discussions
E-mail: marie.bocquillon@umons.ac.be

Éditeur responsable: Lia Machado Fiuza Fialho Experts ad hoc: Cristine Brandenburg et Angélica Silva

\section{Comment citer cet article (ABNT):}

GAUTHIER, Clermont; BISSONNETTE, Steve; BOCQUILLON, Henri. Instruire ou étourdir les élèves? Réflexion critique sur l'idée qu'« il faut varier son enseignement ». Educ. Form., Fortaleza, v. 6, n. 3, e5404, 2021. Disponível em:

https://revistas.uece.br/index.php/redufor/article/view/5404

Educ. Form., Fortaleza, v. 6, n. 3, e5404, set./dez. 2021

DOI: https://doi.org/10.25053/redufor.v6i2.5404

https://revistas.uece.br/index.php/redufor/index 


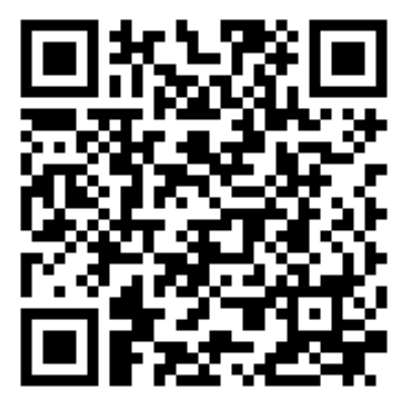

Reçu le 26 avril 2021.

Accepté le 3 mai 2021.

Publié le 2 juin 2021. 